\title{
Learning from Rural Health Innovation
}

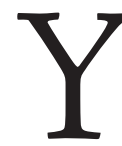

ou know that you're Canadian (or, presumably, Australian, Russian or Brazilian) if you measure distance in hours, not miles, or so the saying goes. The vast distances in sparsely populated landscapes represent substantial challenges for the effective delivery of high-quality care. Resources, specialized care and connections that are readily available in urban areas are in short supply.

But kayaks, snowmobiles and canola are by no means the only innovations to be inspired by the needs of rural Canada. Many creative solutions to healthcare challenges have also emerged beyond big cities. New models of team-based collaborative care, expanded scopes of practice and imaginative uses of telehealth technology to deliver specialized care are just a few examples. There are rich opportunities for sharing innovations and spreading best practices from community to community.

Over time, a number of these innovations have also influenced care in urban settings. For instance, telehealth, once seen as a tool to be used in remote areas, is increasingly common in communities of all sizes, sharing specialized expertise between city-centre hospitals, helping those who are homebound to get the care that they need and more.

This issue of Healthcare Policy/Politiques de Santé has a number of papers that focus on health in rural settings, but their findings are relevant in a range of contexts and challenges - access to care, appropriate program planning and effective health human resources management. Other papers are also broad-based, with findings that can be applied across a number of settings. Michael Law discusses the implications of approaches to generic drug pricing; Clare Liddy and colleagues address practice facilitation programs and Mohammed Al-Hamdani presents the results of new research into plain packaging for cigarettes, to name just a few.

I hope that you will find much food for thought, as well as inspiration for policy and practice improvement, in the journal's pages. 


\section{Tirer leçon de l'innovation en santé rurale}

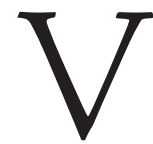

ous savez que vous êtes Canadien (et probablement Australien, Russe ou Brésilien) si vous mesurez les distances en heures et non en kilomètres, selon le dicton. Les vastes distances dans les paysages peu peuplés représentent un défi de taille pour la prestation efficace de soins de première qualité. Les ressources, les soins spécialisés et la proximité, qui sont immédiats dans les zones urbaines, sont plus rares en milieu rural.

Le kayak, la motoneige et le canola ne sont certes pas les seules innovations qui ont été inspirées par les besoins du Canada rural. Plusieurs réponses créatives aux défis touchant les soins de santé ont émergées au-delà des grandes villes. De nouveaux modèles de soins collaboratifs en équipe, des champs d'activités étendus et l'emploi imaginatif des technologies de télésanté pour offrir des soins n'en sont que quelques exemples. Il s'agit là d'une occasion idéale pour partager les innovations et répandre les pratiques exemplaires d'une communauté à l'autre.

Au cours du temps, plusieurs de ces innovations ont également influencé des établissements urbains. Par exemple, la télésanté, auparavant conçue pour les zones rurales, est de plus en plus en vogue dans les communautés de toutes tailles, notamment en permettant le partage de l'expertise entre hôpitaux urbains, ce qui permet d'offrir les soins nécessaires aux personnes confinées à domicile.

Ce numéro de Politiques de Santé/Healthcare Policy présente des articles qui portent sur la santé en milieu rural, mais leurs résultats sont pertinents pour une vaste gamme de contextes et de défis - l'accès aux services, la planification de programme et la gestion efficace des ressources humaines en santé. D'autres articles ont une vaste portée et leurs résultats peuvent sappliquer à de nombreux types d'établissements. Michael Law parle des répercussions de la démarche quant aux prix des médicaments génériques, Clare Liddy et ses collaborateurs abordent la question des programmes de facilitation de la pratique et Mohammed Al-Hamdani présente les résultats d'une nouvelle recherche sur l'emballage neutre des paquets de cigarettes, pour n'en nommer que quelques-uns.

J'espère que, dans les pages de cette revue, vous trouverez matière à alimenter vos réflexions, de même que l'inspiration nécessaire pour améliorer les politiques et la pratique. 